Original Article - Clinical Science

\title{
Effect of phacoemulsification cataract surgery on intraocular pressure in early glaucoma: A prospective multi-site study
}

Ayub Qassim MBBS, ${ }^{* 1}$ Mark J. Walland FRANZCO, ${ }^{2}$ J ohn Landers FRANZCO PhD, ${ }^{1}$ Mona Awadalla MBBS PhD, ${ }^{1}$ Thi Nguyen BMSc MOptom, ${ }^{1}$ Jason Loh BHSc MOrth, ${ }^{1}$ Angela M Schulz PhD, ${ }^{3}$ Bronwyn Ridge BA, ${ }^{1}$ Anna Galanopoulos FRANZCO, ${ }^{4}$ Ashish Agar FRANZCO PhD, ${ }^{5}$ Alex W Hewitt FRANZCO PhD, ${ }^{6}$ Stuart L Graham FRANZCO PhD, ${ }^{3}$ Paul R Healey FRANZCO PhD, ${ }^{7}$ Robert J. Casson FRANZCO DPhil ${ }^{8}$ and J amie E. Craig MBBS DPhil ${ }^{1}$

1. Department of Ophthalmology, Flinders University, Flinders Medical Centre, Bedford Park, Australia.

2. Royal Victorian Eye and Ear Hospital, Melbourne, Australia.

3. Faculty of Medicine and Health Sciences, Macquarie University, Australia

4. South Australian Institute of Ophthalmology, Royal Adelaide Hospital, Adelaide, Australia

5. Department of Ophthalmology, Prince of Wales Hospital, Randwick, Australia

6. Menzies Institute for Medical Research, University of Tasmania, Australia.

7. Centre for Vision Research, Westmead Institute for Medical Research, University of Sydney, Australia

8. South Australian Institute of Ophthalmology, University of Adelaide, Adelaide, Australia

This is the author manuscript accepted for publication and has undergone full peer review but has not been through the copyediting, typesetting, pagination and proofreading process, which may lead to differences between this version and the Version of Record. Please cite this article as doi: $10.1111 /$ ceo.13724

This article is protected by copyright. All rights reserved. 
Correspondence: $\operatorname{Dr}$ Ayub Qassim, Department of Ophthalmology, Flinders University, Bedford Park, Australia. E-mail: ayub.qassim@flinders.edu.au

Level 2 Car Park Tenancies, 1 Flinders Drive, Bedford Park, SA, 5042, Australia

Short running title: Phacoemulsification surgery in glaucoma

Received 18 October 2019; accepted 26 J anuary 2020

Funding sources / Financial disclosure: The work was funded by National Health and Medical Research Council (NHMRC grant 1048037). AQ is supported by an Avant scholarship. JEC is an NHMRC Practitioner Fellow.

Conflict of interest: None 


\section{ABSTRACT}

I mportance: Cataract and primary open angle glaucoma (POAG) commonly coexist, and cataract surgery is thought to reduce intraocular pressure (IOP), the major modifiable risk factor of POAG.

Background: Previous studies exploring the effect of cataract surgery on IOP are limited by retrospective design, lack of a control group, medication use and washout, and loss to follow-up.

Design: Prospective, multicentre, matched case-control Australian study.

Participants: 171 eyes of 108 POAG patients who underwent cataract surgery, matched to 171 control eyes.

Methods: Serial longitudinal IOP measurements was compared before and after cataract surgery, and relative to the controls. A mixed-effect model was used for the longitudinal data.

Main Outcome Measures: Change in IOP.

Results: The mean follow-up time was 4.8 (1.4) years. Cataract surgery reduced mean IOP by $2.22 \mathrm{mmHg}$ (95\% confidence interval [Cl]: 1.93-2.52 mmHg, $\mathrm{P}<0.001$ ) with 59 eyes (34\%) achieving at least $3 \mathrm{mmHg}$ reduction. Compared to matched controls, the mean reduction in IOP was $1.75 \mathrm{mmHg}$ (95\% Cl $1.15-2.33 \mathrm{mmHg}$; $\mathrm{P}<0.001)$. Higher preoperative IOP and being on fewer topical glaucoma medications preoperatively were strongly predictive of a larger IOP reduction in a multivariable model. Anterior chamber depth was not associated with IOP reduction. Eyes with preoperative IOP $\geq 24 \mathrm{mmHg}$ had a mean IOP reduction of $4.03 \mathrm{mmHg}$ with $81 \%$ experiencing at least $3 \mathrm{mmHg}$ reduction. Sub-analysis of medication naïve and pseudoexfoliation patients showed similar results.

Conclusions and Relevance: Cataract surgery has a confirmed effect in reducing IOP in a "real world" setting of early glaucoma patients. 
Keywords: Open angle glaucoma, Cataract surgery, Phacoemulsification, Cataract, Biometry 


\section{I NTRODUCTI ON}

Glaucoma is a progressive optic neuropathy characterised by accelerated loss of retinal ganglion cells, leading to visual field loss. ${ }^{1}$ The most common subtype of glaucoma is primary open angle glaucoma (POAG) in which the iridocorneal angle is unobstructed by the iris and there is no secondary cause of intraocular pressure (IOP) elevation. The prevalence of POAG and cataract are strongly associated with older age, and thus both conditions commonly coexist. ${ }^{2,3}$ Glaucoma and cataract represent the second and third most common causes of blindness in Australia, respectively. ${ }^{4}$ Cataract is the commonest cause of non-refractive error-related lowvision in Australia and worldwide. ${ }^{4}$

Currently, lowering IOP is the only demonstrably therapeutic treatment for POAG. Both topical medical therapy and selective laser trabeculoplasty (SLT) are effective and commonly used to treat POAG. ${ }^{5,6}$ Filtration surgery is a more widely effective means of lowering IOP but carries higher morbidity compared to medical or laser therapy and thus is commonly reserved for more recalcitrant disease not responding to other therapies. ${ }^{7,8}$

While cataract surgery is not a procedure for IOP lowering in open angle glaucoma, several studies have reported a reduction of IOP following cataract surgery in patients with open angles. ${ }^{9,10}$ A recent meta-analysis of the studies on the effects of phacoemulsification on IOP has identified the lack of a control group, medication use and washout, and the loss to follow-up as sources of bias which potentially undermine certain conclusions in the reported literature. ${ }^{11}$ In this study, we aim to address these issues and characterise the effects of cataract surgery on IOP in a large prospectively monitored early glaucoma cohort with pragmatic study design 
across multiple clinics in Australia. In the following text, cataract surgery refers to phacoemulsification with posterior chamber intraocular lens insertion.

\section{METHODS}

\subsection{Study participants}

This study cohort formed a sub-population of PROGRESSA study (Progression Risk Of Glaucoma; RElevant SNPs with Significant Association), an Australia-wide multicentre prospective cohort study of progression risk in glaucoma.

Participants were enrolled if they had optic nerve head appearance suspicious or probable for early stage glaucoma based on the disk damage likelihood scale (DDLS) ${ }^{12}$ with Humphrey visual field mean deviation better than $-6 \mathrm{db}$ (Humphrey Field Analyzer; Carl Zeiss Meditec; Dublin, CA). Patients with angle closure on gonioscopy, secondary causes of elevated IOP or other ophthalmic conditions causing reduced visual acuity were excluded. Patients were reviewed 6-monthly with complete ophthalmic examination including visual acuity and IOP measurements using Goldmann Applanation Tonometry (GAT). Dilated fundoscopy and central corneal thickness (CCT) measurement by pachymetry (Pachmate DGH55; DGH Technology Inc, Exton, PA) were performed annually. If a participant had glaucoma filtration surgery, then only those visits prior to this operation were included in the analysis.

Eligible participants were those who had undergone cataract surgery via phacoemulsification with intraocular lens implant in either eye during their period of longitudinal monitoring, and had at least one visit 6-months post procedure. The indication for surgery was visually significant cataract interfering with vision. Surgical technique and IOP management was at the discretion of the treating glaucoma specialist. Case notes were then reviewed to record the pre-treatment maximum 
IOP, and the preoperative ocular biometry: anterior chamber depth and axial length (IOL Master, Carl Zeiss, Germany).

A matched sample of the PROGRESSA cohort who had not undergone any ocular surgery during their follow-up were selected as controls. These were enrolled and followed up under the same protocol as outlined above. To account for confounding factors that could affect IOP measurements over time, the control group were matched to the cases (who underwent cataract surgery) by age, gender, duration of study follow-up, baseline IOP at enrolment, and treatment intensity during followup.

Formal participant information was provided and informed consent was obtained from all study participants. The study was approved by the The Southern Adelaide Clinical Human Research Ethics Committee (SACHREC) and adheres to the tenets of the Declaration of Helsinki.

\subsection{Statistical analysis}

All statistical analyses were performed using R (version 3.5.1, RCore Team, Austria). ${ }^{13}$ Both eyes, if applicable, were included in the study. Controls were matched by dissimilarity matrix calculation using cluster (version 2.0.7.1) and e1071 (version 1.7.0.1) packages in R. ${ }^{14,15}$ Analysis of variance between the cases and controls was done using Kruskal-Wallis test for continuous variables and Chi-squared test for categorical variables. To account for the correlated nature of these measurements, we used a mixed-effects linear model with random intercepts per patient. ${ }^{16}$ Longitudinal IOP measurements from each visit was used in the primary analysis; within-eye correlation was adjusted for using two-level random intercepts per patient then per eye. ${ }^{16}$ In the case-control analysis, we calculated the mean difference between all the IOP measurements before and after cataract surgery for 
the cases. For the controls, the median visit per patient was used as the point in time to calculate the "change in IOP". To account for the random effects of the clinic sites, we included the recruitment and follow-up location as another random intercept in the model. Models were fitted using Ime4 package (version 1.1.20), ${ }^{17}$ and hypothesis testing of the model was done using Satterthwaite's degrees of freedom method. The pseudo- $R^{2}$ of the mixed-effects model was calculated based on the fixed-effects only as described by Nakagawa et al. ${ }^{18}$ The cut-off of statistical significance (alpha level) was set at $\mathrm{P}=0.05$.

\section{RESULTS}

In total, 171 eyes of 108 patients from 8 clinics across Australia met the case eligibility criteria for this study. All cases were enrolled in PROGRESSA and underwent cataract surgery electively during routine follow-up. The mean (SD) age of the patients at the time of cataract extraction was 72.8 (6.8) years. Cases had clinical data recorded for 3.9 (3.4) years before the operation with a mean follow-up time of 2.7 (1.7) years post procedure. The majority of our patients had at least one year of follow-up ( $n=145,85 \%$ ). Follow-up rates at 18 and 24 months post procedure were $73 \%(n=125)$ and $59 \%(n=100)$ respectively. Five eyes $(2.9 \%)$ underwent trabeculectomy surgery at a later date due to progressive glaucoma not responding to medical treatment. IOP measurements following the trabeculectomy were not included in the analysis.

For our control group, we matched 171 eyes of 144 patients enrolled in PROGRESSA and follow-up by the same protocol as the cases, who have not undergone cataract surgery. As cataract surgery was indicated for visually significant cataracts, there was a significant difference in the best corrected visual acuity (BCVA) between the cases (prior to cataract surgery) and the controls (median [IQR] BCVA 6/9.5 [4.5] vs 
6/7.6 [3.5] respectively; $\left.P=3.3 \times 10^{-8}\right)$. The distribution of the cataract density of the cases and the controls using The Lens Opacities Classification System III is presented in Supplementary Figure 1. Detailed clinical and demographic characteristics of the study cohort is summarised in Table 1.

Table 1: Clinical and demographic characteristics of study participants

\begin{tabular}{|c|c|c|c|}
\hline Characteristics & Cases & Controls & $\mathbf{P}$ \\
\hline Number: eyes / patients & $171 / 108$ & $171 / 144$ & \\
\hline Gender, male (\%) & $56(51.9)$ & $69(47.9)$ & 0.62 \\
\hline Age at recruitment, years & $69.2(6.8)$ & $69.0(6.5)$ & 0.93 \\
\hline Study follow-up duration, years & $4.8(1.4)$ & $4.43(1.5)$ & 0.05 \\
\hline $\begin{array}{l}\text { Number with } 5 \text { year follow-up, } \\
\text { patients }(\%)\end{array}$ & $55(50.9)$ & $58(40.3)$ & 0.12 \\
\hline $\begin{array}{l}\text { Pseudoexfoliation syndrome, } \\
\text { patients }(\%)\end{array}$ & $5(4.6)$ & $4(2.8)$ & 0.66 \\
\hline Central corneal thickness, $\mu \mathrm{m}$ & $546(35)$ & $538(31)$ & 0.05 \\
\hline IOP at recruitment, $\mathrm{mmHg}$ & $16.7(4.2)$ & $16.5(3.6)$ & 0.90 \\
\hline Maximum recorded IOP, mmHg & $20.6(5.8)$ & $20.4(5.2)$ & 0.88 \\
\hline Vertical cup-to-disc ratio & $0.71(0.12)$ & $0.67(0.13)$ & 0.01 \\
\hline Visual field mean deviation, $\mathrm{dB}$ & $-2.72(3.95)$ & $-2.38(3.14)$ & 0.65 \\
\hline Number of glaucoma medications & $1.0(1.0)$ & $0.9(0.9)$ & 0.52 \\
\hline $\begin{array}{l}\text { Selective laser trabeculoplasty, } \\
\text { eyes }(\%)\end{array}$ & $\begin{array}{r}57 \\
(34.1)\end{array}$ & $\begin{array}{r}43 \\
(25.1)\end{array}$ & 0.09 \\
\hline $\begin{array}{l}\text { Number of treatment naive } \\
\text { patients }(\%)\end{array}$ & $27(25.0)$ & $43(29.9)$ & 0.47 \\
\hline
\end{tabular}

Values displayed are mean (standard deviation) for continuous variables and $\mathrm{N}(\%)$ for categorical variables. $\mathrm{P}$ values represent the statistical significance of the analysis 
of variance for continuous variables (Kruskal-Wallis test) or Chi-squared test for categorical variables.

IOP: intraocular pressure.

Using the longitudinal data of all IOP measurements, cataract surgery reduced the mean IOP by $2.22 \mathrm{mmHg}$ (95\% confidence interval [Cl]: $1.93-2.52 \mathrm{mmHg}$; $\mathrm{P}<$ $\left.2 \times 10^{-16}\right)$. The effect was most pronounced in the first 18 months after cataract surgery then diminished to some extent although remaining below pre-operative mean measurements (Figure 1A). The magnitude of reduction differed between eyes, with 59 eyes (34\%) achieving at least $3 \mathrm{mmHg}$ reduction within two years of cataract surgery, and 34 eyes $(20 \%)$ having no, or a positive, change in IOP. A breakdown of the change in IOP per eye within two years of the cataract surgery is summarised in Table 2 and Figure 2. Ongoing glaucoma management was the discretion of the treating clinicians and was individualised to each patient. Thus, only 5 eyes (3\%) stopped at least one glaucoma medication post cataract surgery. Escalation in medical treatment was similar between the cases (29 eyes) and controls (27 eyes; $P=0.9$ ). 


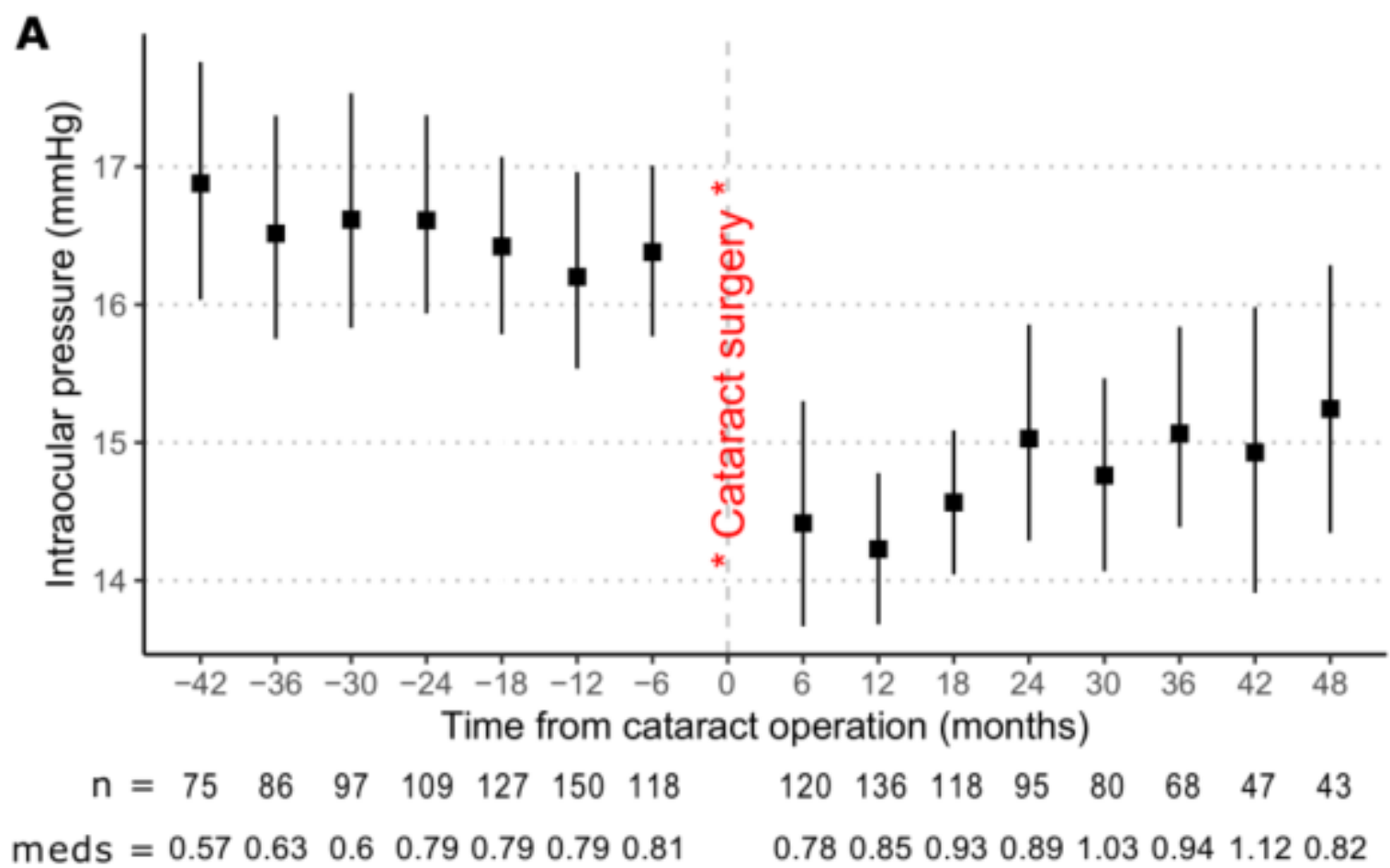

B

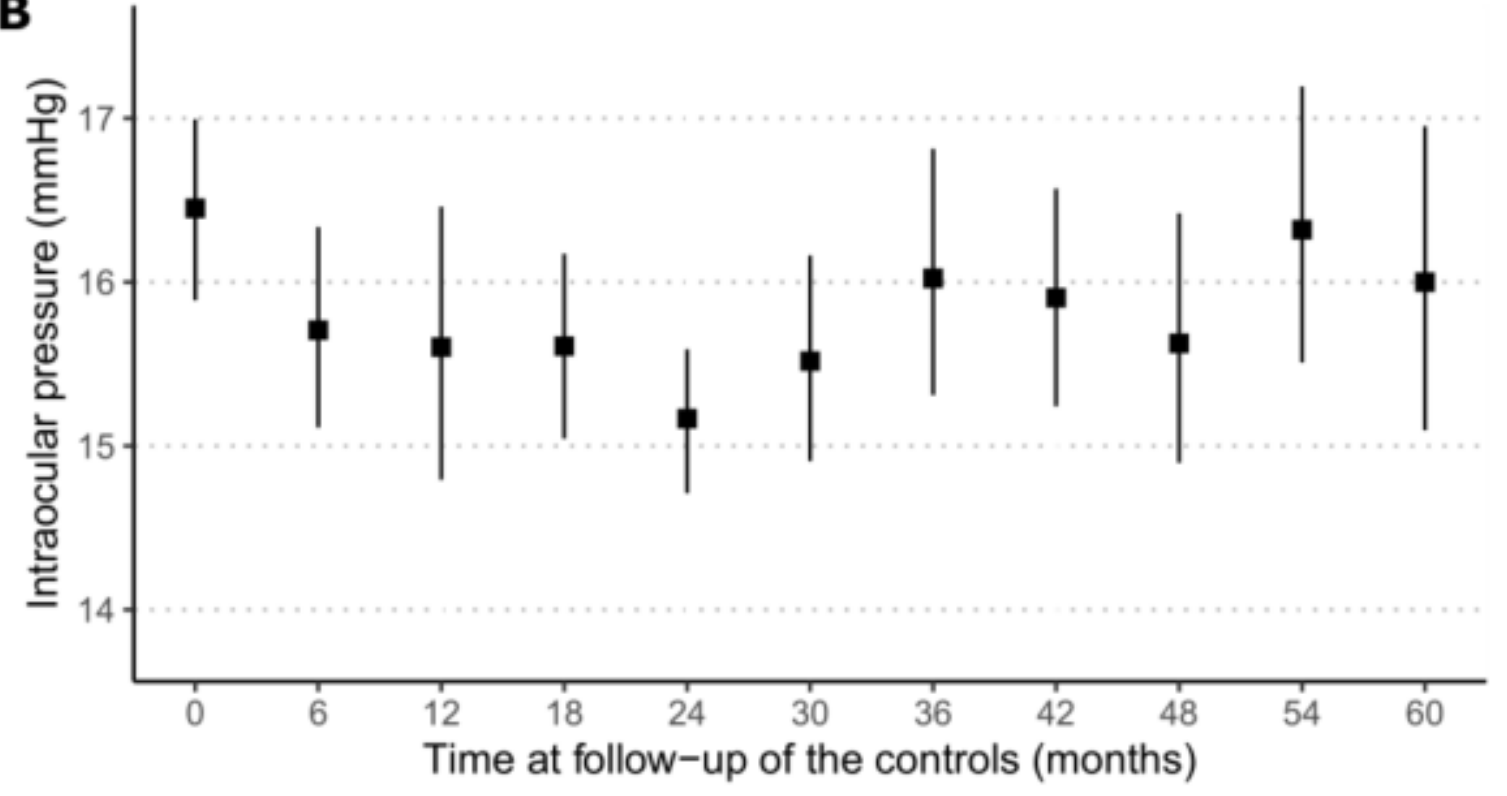

\begin{tabular}{|c|c|c|c|c|c|c|c|c|c|}
\hline $\mathrm{n}=171$ & 112 & 58 & 148 & 146 & 135 & 121 & 114 & 102 & 104 \\
\hline meds $=0.74$ & 0.83 & 0.77 & 0.78 & 0.82 & 0.79 & 0.89 & 0.84 & 0.93 & 0.9 \\
\hline
\end{tabular}

This article is protected by copyright. All rights reserved. 
Figure 1: Longitudinal change in intraocular pressure (IOP) after cataract surgery (A) in the cases $n=171$, and (B) in the control group $n=171$ eyes. The square dot represents the mean IOP at each time point, and the error bars represent the $95 \%$ confidence interval of the mean. The number of eyes with IOP data recorded and the mean number of topical glaucoma medications at each time point is reported below each figure.

Table 2: Number and percent of eyes with the corresponding two-year mean change in intraocular pressure following cataract surgery

\begin{tabular}{|c|c|c|}
\hline $\begin{array}{l}\text { Two-year absolute } \\
\text { change in I OP after } \\
\text { cataract surgery }\end{array}$ & $\begin{array}{r}\text { Number of } \\
\text { eyes }\end{array}$ & Percent of eyes \\
\hline At least $-6 \mathrm{mmHg}$ & 7 & $4.1 \%$ \\
\hline-6 up to $-5 \mathrm{mmHg}$ & 11 & $6.4 \%$ \\
\hline-5 up to $-4 \mathrm{mmHg}$ & 15 & $8.8 \%$ \\
\hline-4 up to $-3 \mathrm{mmHg}$ & 19 & $11 \%$ \\
\hline-3 up to $-2 \mathrm{mmHg}$ & 33 & $19.3 \%$ \\
\hline-2 up to $-1 \mathrm{mmHg}$ & 23 & $13.5 \%$ \\
\hline-1 up to $0 \mathrm{mmHg}$ & 29 & $17 \%$ \\
\hline No or positive change & 34 & $19.9 \%$ \\
\hline
\end{tabular}

IOP: intraocular pressure. 


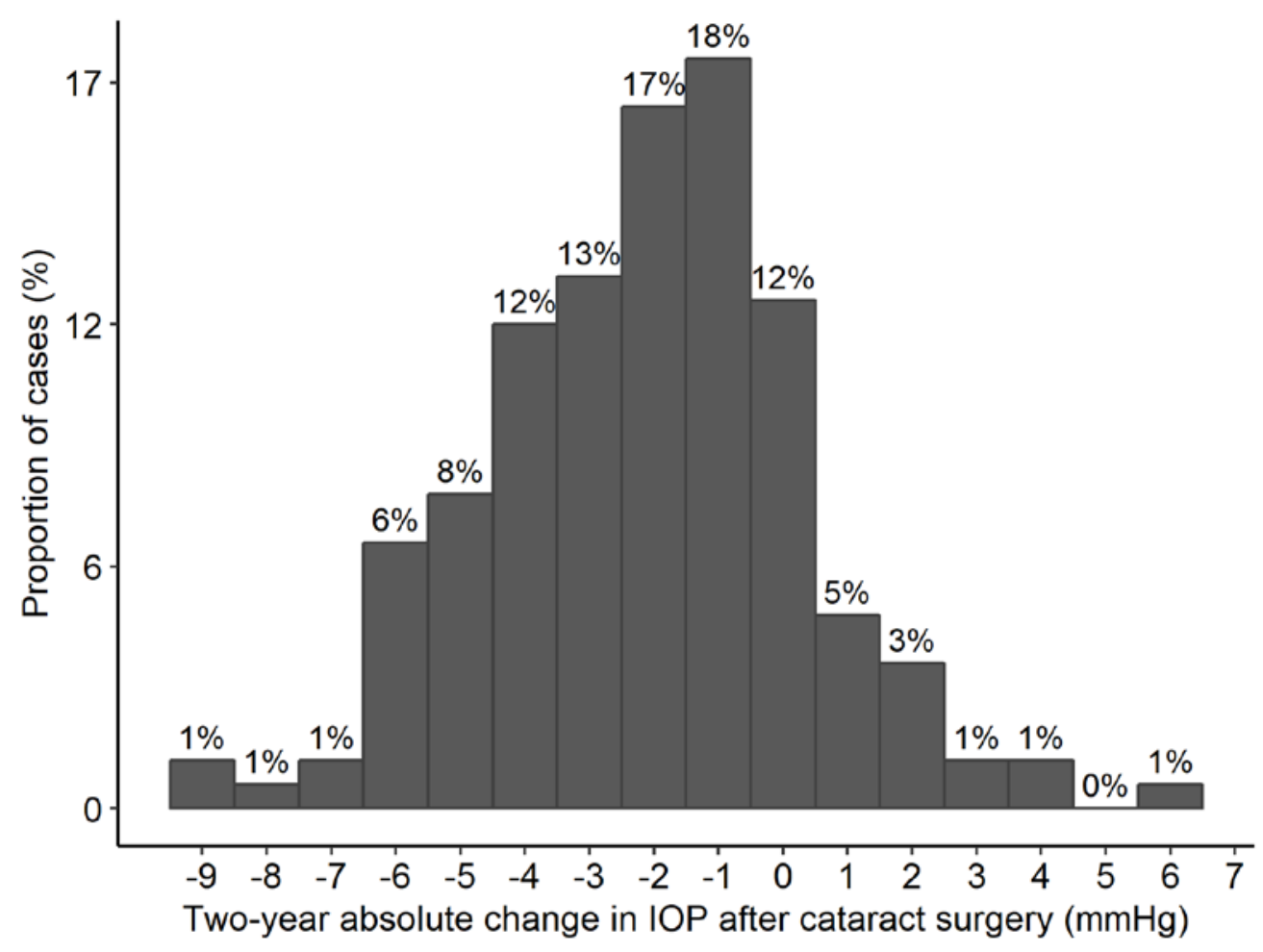

Figure 2: Histogram of the absolute change in intraocular pressure (IOP) within 2 years after cataract surgery in the PROGRESSA cohort ( $\mathrm{N}=171$ eyes). IOP: intraocular pressure.

To further isolate the effect of cataract surgery from the change of IOP over time, we conducted a case-control analysis, comparing the change in IOP in those who underwent cataract surgery (i.e. the group reported above) to matched controls (no cataract surgery; $\mathrm{N}=171$ eyes). The controls had similar age, gender, IOP at enrolment, duration of follow-up, and medical treatment compared to the cases (Table 1). Compared to the controls, and accounting for the intereye correlation of IOP and the clinic site as a random effect, cataract surgery reduced IOP by 1.75 $\mathrm{mmHg}\left(95 \% \mathrm{Cl} 1.15-2.33 \mathrm{mmHg} ; \mathrm{P}=1.6 \times 10^{-8}\right)$.

To identify predictors of the change in IOP for inclusion in a multiple variable model, we conducted univariate linear regressions with the following variables: gender, age 
at operation, maximum recorded IOP prior to the operation, baseline IOP at study enrollment, central corneal thickness, axial length, anterior chamber depth, history of pseudoexfoliation syndrome, and the number of topical glaucoma medications prior to the operation (Supplementary Table 1). Variables with P-values $<0.2$ were then used in a multiple variable model. When the maximum recorded IOP was limited to two years prior to cataract surgery, the strength of the association and the goodness-of-fit of the change in IOP improved significantly with $23 \%$ of the variance in the postoperative IOP change explained compared to $7 \%$ when the all-time maximum IOP was used. We have thus included the two-year maximum in the multiple variable model. Interestingly, preoperative anterior chamber depth and axial length were not associated with the change in IOP postoperatively (Supplementary Figure 2).

In the multiple variable model, elevated preoperative IOP was strongly predictive of a larger reduction in IOP post cataract surgery (Table 3). For each $1 \mathrm{mmHg}$ higher maximum preoperative IOP (in the preceding 2 years), there was an estimated reduction of $0.33 \mathrm{mmHg}(95 \% \mathrm{Cl}: 0.23-0.42 \mathrm{mmHg}$ ) of IOP post cataract surgery ( $\mathrm{P}$ $\left.=1.4 \times 10^{-10}\right)$. The number of glaucoma medications preoperatively was inversely associated with the change in IOP. That is, patients who were on more intensive glaucoma medical therapy experienced a lower reduction in IOP than those with fewer topical glaucoma drops (Supplementary Figure 3). This may suggest that cataract surgery is subject to the so-called 'law of diminishing returns', in producing a lesser yield of IOP-lowering benefit in those already on treatment. ${ }^{19}$ Finally, male participants experienced an estimated $1 \mathrm{mmHg}(95 \% \mathrm{Cl}: 0.30-1.7 \mathrm{mmHg})$ greater reduction in IOP than females $\left(P=7.5 \times 10^{-3}\right)$ 
Table 3: Multiple variable model of clinical predictors of IOP reduction following cataract surgery.

\begin{tabular}{|c|c|c|c|}
\hline Variable & Estimate & $\begin{array}{l}\text { Standard } \\
\text { error }\end{array}$ & P-value \\
\hline $\begin{array}{l}\text { Number of glaucoma medications } \\
\text { preoperatively }\end{array}$ & 0.77 & 0.21 & $3.0 \times 10^{-4}$ \\
\hline $\begin{array}{l}\text { Maximum IOP in the last } 2 \text { years } \\
\text { prior to cataract operation }\end{array}$ & -0.33 & 0.048 & $1.4 \times 10^{-10}$ \\
\hline Baseline study IOP & 0.073 & 0.037 & 0.05 \\
\hline Gender (male) & -1.01 & 0.37 & $7.5 \times 10^{-3}$ \\
\hline
\end{tabular}

Results are the coefficients of a linear mixed-effects model with the post cataract change in IOP as the response and the variables as fixed predictors. The model random effects were the patients (to account for the intereye correlation) and clinic site. Pseudo-R-squared (fixed effects only) $=35 \%$.

IOP: intraocular pressure.

We further examined the impact of high preoperative IOP on the expected postoperative reduction in pressure (Figure 3). Eyes with 2-year preoperative maximum IOP of at least $24 \mathrm{mmHg}(\mathrm{N}=16)$ had a mean IOP reduction of 4.03 (3.27) $\mathrm{mmHg}$ in the first 2 years, with 13 of the eyes (81\%) experiencing at least 3 $\mathrm{mmHg}$ reduction. The outlier in the top right of Figure 3 was a patient with POAG and a persistently high IOP despite maximal medical therapy (4 medications). The cataract operation was done in anticipation of a trabeculectomy which was done 6 months later (no post-trabeculectomy IOP data was included in the analysis). 


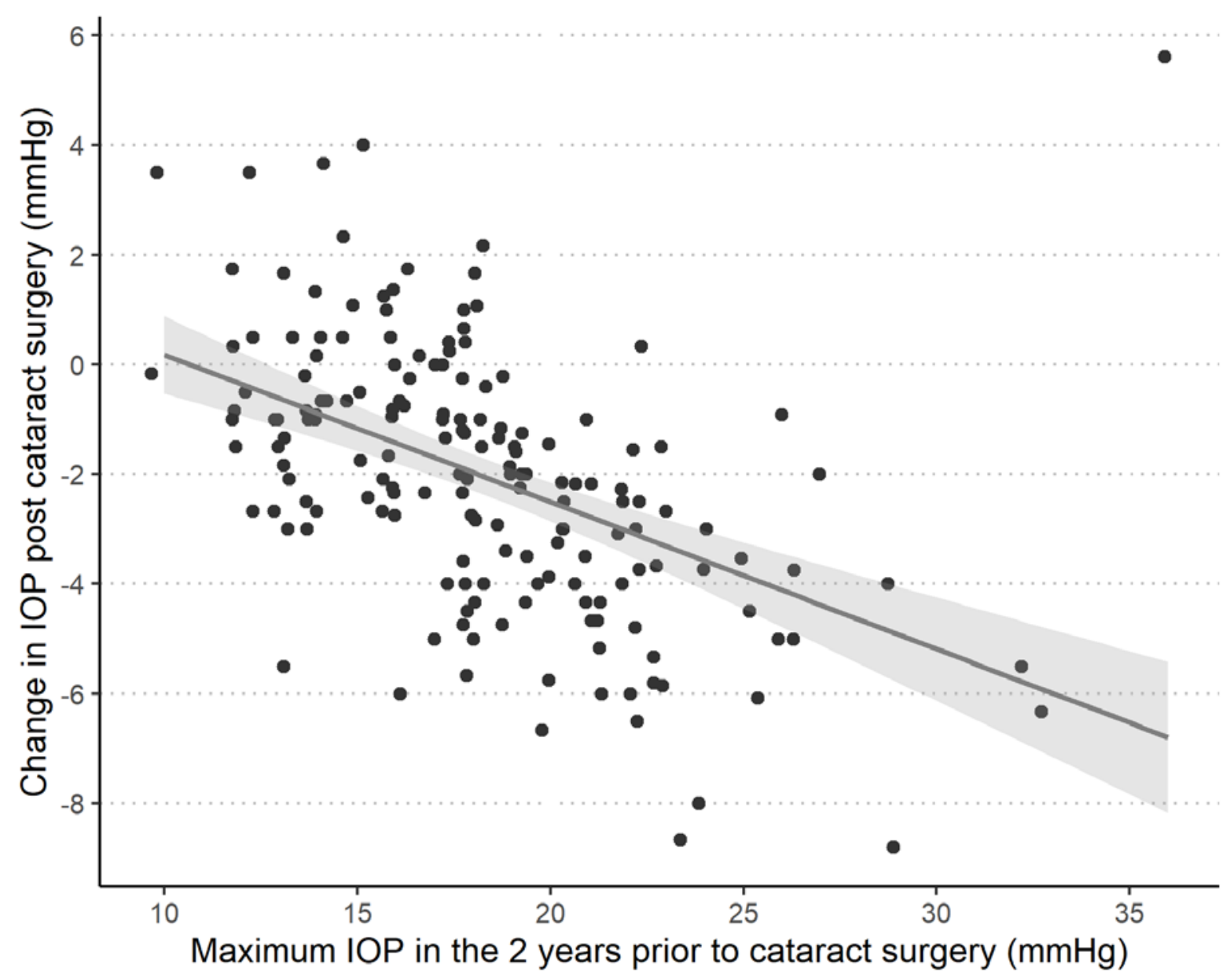

Figure 3: Observed difference in the mean intraocular pressure (IOP) within two years post cataract surgery. Each point represents a study eye. The grey line is the line of best fit with the 95\% confidence interval shaded in light grey (P-value for trend $\left.=3.3 \times 10^{-10}\right)$.

IOP: intraocular pressure.

To improve the generalisability of our results we performed various sensitivity analyses. Topical medications used to treat glaucoma will invariably affect the IOP changes measured during follow-up. To account for this important covariate, we performed a subgroup analysis on medication naive participants - i.e. those who have not been on any topical ocular hypotensive drops during the study period ( $\mathrm{N}=$ 
43 eyes). In this subgroup, cataract surgery was associated with a mean reduction of IOP of $2.4 \mathrm{mmHg}\left(95 \% \mathrm{Cl} 1.9-2.9 \mathrm{mmHg}\right.$; $\left.<2 \times 10^{-16}\right)$. Only one eye had prior peripheral iridotomy and had a mean reduction of $3.8 \mathrm{mmHg}$. Exclusion of this case did not change the results. Eyes with pseudoexfoliation syndrome may experience IOP reduction via a different mechanism than POAG eyes. 7 eyes had pseudoexfoliation syndrome and in this subgroup with a mean IOP reduction of 2.7 $\mathrm{mmHg}\left(95 \% \mathrm{Cl} 1.2-4.1 \mathrm{mmHg} ; \mathrm{P}=4.7 \times 10^{-4}\right)$. This change in IOP was no statistically significantly different than the rest of the study cohort $(P=0.6)$.

\section{DISCUSSION}

This study demonstrated the effects of cataract surgery on intraocular pressure (IOP) in early open angle glaucoma patients in a "real world" setting from 8 clinics in Australia. Patients were enrolled in the study for glaucoma monitoring, and the treatment of cataract was based on symptoms and clinical assessment. We demonstrated a reduction in IOP after cataract surgery that was statistically significant, which persisted for the duration of follow-up, although was most pronounced in the first 18 months. A third of the cases had at least $3 \mathrm{mmHg}$ of mean IOP reduction in 2 years. Patients with higher IOP before the operation, and those on fewer glaucoma medications had a larger reduction in IOP. The cataract surgery in this cohort was not aimed to modify the glaucoma medical therapy, and we did not observe a significant number of patients who had their glaucoma medications reduced.

Our findings are consistent with previously published studies on the effect of cataract surgery on IOP in glaucoma. ${ }^{9-11,20}$ In a study of 55 eyes with POAG, Shingleton et al. reported a mean reduction of $1.4 \pm 3.3 \mathrm{mmHg}$ at the three-year follow-up post cataract surgery, which has persisted at their last follow-up 
appointment (mean of 5 years). ${ }^{16}$ Hayashi et al. observed a mean reduction of $4.3 \pm$ $4.2 \mathrm{mmHg}$ in 68 eyes with POAG at 1 year post cataract surgery, which has persisted for patients followed up to 2 years. ${ }^{9}$ This larger effect size may be attributable to the relatively higher preoperative IOP in this study (mean of $20.7 \mathrm{mmHg}$ ). Other studies have demonstrated a comparable reduction in ocular hypertensives and normal eyes without glaucoma.10,21,22 Cataract surgery may therefore be a useful treatment option in the management in early high tension glaucoma patients with comorbid cataracts.

Microinvasive glaucoma surgery (MIGS) has recently been introduced as a treatment option for early glaucoma. ${ }^{23}$ The currently available devices are usually inserted during cataract surgery. The effect of combined MIGS and cataract surgery relative to cataract surgery alone has been reported in several studies. ${ }^{24-28}$ Of note, these comparative studies only recruited eyes with high tension glaucoma, where our study and others show that cataract removal is most likely to be efficacious, and the results are often reported after medication washout, which whilst valid as a trial tool, is less relevant in a clinical scenario where IOP reduction from the treated IOP is the therapeutic aim. ${ }^{11,20}$

For instance, Craven et. al. reported that cataract surgery alone reduced IOP by 7.5 $\mathrm{mmHg}$ at 24 months post procedure. ${ }^{25} \mathrm{In}$ this high tension POAG cohort, the mean pre-procedure IOP was $25.2 \mathrm{mmHg}$ after medication washout period, thus regression to the mean is expected. Similarly, Pfeiffer et. al. reported $9.2 \mathrm{mmHg}$ IOP reduction at 12 months post cataract surgery in their high tension POAG cohort (mean baseline IOP of $26.6 \mathrm{mmHg}$ ). ${ }^{27}$ The effect size has diminished at 24 months to a net IOP reduction of $7.4 \mathrm{mmHg}$, consistent with our results and others that the effect of cataract surgery on IOP may reduce in some patients over time. ${ }^{22,27}$ 
The mechanism of the IOP reduction seen after cataract surgery may partly be related to the anatomical changes due to the crystalline lens removal. Issa et al. have developed an index for predicting the reduction in IOP post cataract surgery in non-glaucomatous eyes. ${ }^{29}$ Elevated preoperative IOP and shallower anterior chamber depth (ACD) were both predictive of greater IOP reduction postoperatively, consistent with the experience in angle closure eyes. ${ }^{29}$ The ratio of preoperative IOP to preoperative ACD was positively correlated to the magnitude of IOP reduction and there was at least $4 \mathrm{mmHg}$ IOP reduction in those with a ratio higher than 7.0.29 The authors believe that any role of ACD is likely related to the effect of lens thickness on this parameter. It should be noted that gonioscopy was not performed on the participants of this study. In our study, all participants had open angles by gonioscopy and we found no correlation between the ACD or axial length and the change in IOP. This suggests that ACD is not a crucial predictor of IOP reduction post cataract surgery in those with open angles by clinical examination.

Using anterior segment optical coherence tomography in open angle glaucoma patients undergoing cataract operation, Lin et al. reported the angle-opening distance, iris thickness, iris area and the lens vault (the area between the anterior pole of the phakic lens and the line between the two scleral spurs) were associated with greater IOP reduction post surgery, ${ }^{30}$ although other studies have struggled to use such parameters to predict IOP lowering after cataract surgery. ${ }^{31}$ Lensectomy is also speculated to possibly improve aqueous drainage by providing posterior traction on the ciliary body and scleral spur resulting in widening of the trabecular meshwork and Schlemm's canal. ${ }^{32}$

In PXF, reversal of anterior chamber shallowing due to zonular laxity as well as anterior chamber lavage of PXF material have been postulated as reasons for greater 
IOP lowering in PXF cataract patients, and one cannot exclude the possibility that such lavage and simple trabecular meshwork stretching may contribute to the transitory nature of the IOP lowering after routine cataract surgery. ${ }^{33}$ Finally, the phacoemulsification ultrasound may improve trabecular meshwork aqueous drainage. DeVience et al. reported that intraoperative phacoemulsification time was associated with greater IOP reduction in normal eyes. ${ }^{34}$ This is supported by an invitro study of phacoemulsification ultrasound on trabecular meshwork cells activating cellular pathways leading to improved aqueous outflow and lower IOP. ${ }^{35}$ This stressmediated pathway may be similar to the effects of laser trabeculoplasty on the trabecular meshwork. ${ }^{35,36}$

The strengths of this study are the prospective follow-up of a large sample size from multiple clinics and specialists. The pragmatic design reflects the current clinical practice in the management of early glaucoma patients with cataracts. We have also used improved statistical modelling that utilises this longitudinal data and adjustments for several covariates including the clinic site. ${ }^{16}$ This study has some limitations. Our cohort represents early open angle glaucoma cases, and thus our results are not directly applicable to normal eyes or those with other forms or degrees of glaucoma. The cataract density and type may be a factor that contributes to the differences observed between the cases and controls. Other treatments were not standardised by protocol, and thus patients were medically treated per their glaucoma specialists. We have performed sub-analysis to address this, with similar findings to our primary analysis.

In this prospectively monitored early open angle glaucoma cohort, cataract surgery has a confirmed effect in reducing intraocular pressure. The magnitude of effect is largest among those with higher pressures and fewer glaucoma medications. The magnitude of the IOP reduction is generally small. It will remain a point of debate as 
to whether this magnitude of reduction is clinically useful in glaucoma care, particularly if as a deliberate surgical intervention for progressive glaucoma. 


\section{REFERENCES}

1. Casson RJ, Chidlow G, Wood JP, Crowston JG, Goldberg I. Definition of glaucoma: clinical and experimental concepts. Clinical \& Experimental Ophthalmology. 2012 May 1;40(4):341-9.

2. McCarty CA, Mukesh BN, Fu CL, Taylor HR. The epidemiology of cataract in Australia. American J ournal of Ophthalmology. 1999 Oct 1; 128(4):446-65.

3. Mitchell P, Smith W, Attebo K, Healey PR. Prevalence of open-angle glaucoma in Australia. The Blue Mountains Eye Study. Ophthalmology. 1996 Oct; 103(10):1661-9.

4. Taylor HR, Keeffe JE, Vu HTV, Wang JJ, Rochtchina E, Mitchell P, et al. Vision Ioss in Australia. The Medical J ournal of Australia. 2005 J un 6; 182(11):565-8.

5. Wong MOM, Lee JWY, Choy BNK, Chan JCH, Lai JSM. Systematic review and meta-analysis on the efficacy of selective laser trabeculoplasty in open-angle glaucoma. Surv Ophthalmol. 2015 Feb;60(1):36-50.

6. Garway-Heath DF, Crabb DP, Bunce C, Lascaratos G, Amalfitano F, Anand N, et al. Latanoprost for open-angle glaucoma (UKGTS): a randomised, multicentre, placebo-controlled trial. The Lancet. 2015 Apr 4; 385(9975): 1295-304.

7. Caprioli J, de Leon JM, Azarbod P, Chen A, Morales E, Nouri-Mahdavi K, et al. Trabeculectomy Can Improve Long-Term Visual Function in Glaucoma. Ophthalmology. 2016 Jan; 123(1):117-28.

8. Feiner L, Piltz-Seymour JR, Collaborative Initial Glaucoma Treatment Study. Collaborative I nitial Glaucoma Treatment Study: a summary of results to date. Curr Opin Ophthalmol. 2003 Apr;14(2):106-11.

9. Hayashi K, Hayashi H, Nakao F, Hayashi F. Effect of cataract surgery on intraocular pressure control in glaucoma patients. Journal of Cataract \& Refractive Surgery. 2001 Nov; 27(11):1779-86.

10. Shingleton BJ, Pasternack JJ, Hung JW, O??Donoghue MW. Three and Five Year 
Changes in Intraocular Pressures After Clear Corneal Phacoemulsification in Open Angle Glaucoma Patients, Glaucoma Suspects, and Normal Patients: J ournal of Glaucoma. 2006 Dec; 15(6):494-8.

11. Masis M, Mineault PJ, Phan E, Lin SC. The role of phacoemulsification in glaucoma therapy: A systematic review and meta-analysis. Survey of Ophthalmology. 2018 Sep 1;63(5): 700-10.

12. Bayer A, Harasymowycz P, Henderer JD, Steinmann WG, Spaeth GL. Validity of a new disk grading scale for estimating glaucomatous damage: correlation with visual field damage. Am J Ophthalmol. 2002 J un; 133(6): 758-63.

13. R Core Team. R: A Language and Environment for Statistical Computing [Internet]. Vienna, Austria: R Foundation for Statistical Computing; 2018. Available from: https://www.R-project.org/

14. Maechler M, Rousseeuw P, Struyf A, Hubert M, Hornik K. cluster: Cluster Analysis Basics and Extensions. 2018.

15. Meyer D, Dimitriadou E, Hornik K, Weingessel A, Leisch F. e1071: Misc Functions of the Department of Statistics, Probability Theory Group (Formerly: E1071), TU Wien [Internet]. 2019. Available from: https://CRAN.Rproject.org/package $=\mathrm{e} 1071$

16. Fan Q, Teo Y-Y, Saw S-M. Application of Advanced Statistics in Ophthalmology. Invest Ophthalmol Vis Sci. 2011 Aug 1;52(9):6059-65.

17. Bates D, Mächler M, Bolker B, Walker S. Fitting Linear Mixed-Effects Models Using Ime4. Journal of Statistical Software [Internet]. 2015 [cited 2019 Feb 20];67(1). Available from: http://www.jstatsoft.org/v67/i01/

18. Nakagawa $S$, Johnson $P C D$, Schielzeth $H$. The coefficient of determination $R 2$ and intra-class correlation coefficient from generalized linear mixed-effects models revisited and expanded. Journal of The Royal Society Interface. 2017 Sep 30;14(134):20170213.

19. Camras CB, Toris CB, Tamesis RR. Efficacy and Adverse Effects of Medications 
Used in the Treatment of Glaucoma: Drugs \& Aging. 1999; 15(5):377-88.

20. Melancia D, Pinto LA, Marques-Neves C. Cataract Surgery and Intraocular Pressure. ORE. 2015;53(3):141-8.

21. Pohjalainen T, Vesti E, Uusitalo RJ, Laatikainen L. Intraocular pressure after phacoemulsification and intraocular lens implantation in nonglaucomatous eyes with and without exfoliation. J Cataract Refract Surg. 2001 Mar;27(3):426-31.

22. Mansberger SL, Gordon MO, Jampel H, Bhorade A, Brandt JD, Wilson B, et al. Reduction in intraocular pressure after cataract extraction: the Ocular Hypertension Treatment Study. Ophthalmology. 2012 Sep; 119(9): 1826-31.

23. Conlon R, Saheb H, Ahmed IIK. Glaucoma treatment trends: a review. Canadian Journal of Ophthalmology. 2017 Feb 1;52(1):114-24.

24. Fernández-Barrientos Y, García-Feijoó J, Martínez-de-la-Casa JM, Pablo LE, Fernández-Pérez C, García Sánchez J. Fluorophotometric study of the effect of the glaukos trabecular microbypass stent on aqueous humor dynamics. Invest Ophthalmol Vis Sci. 2010 J ul; 51(7):3327-32.

25. Craven ER, Katz LJ, Wells JM, Giamporcaro JE, iStent Study Group. Cataract surgery with trabecular micro-bypass stent implantation in patients with mild-tomoderate open-angle glaucoma and cataract: two-year follow-up. J Cataract Refract Surg. 2012 Aug;38(8):1339-45.

26. Fea AM. Phacoemulsification versus phacoemulsification with micro-bypass stent implantation in primary open-angle glaucoma: Randomized double-masked clinical trial. Journal of Cataract \& Refractive Surgery. 2010 Mar 1;36(3):40712.

27. Pfeiffer N, Garcia-Feijoo J, Martinez-de-la-Casa JM, Larrosa J M, Fea A, Lemij H, et al. A Randomized Trial of a Schlemm's Canal Microstent with Phacoemulsification for Reducing Intraocular Pressure in Open-Angle Glaucoma. Ophthalmology. 2015 J ul; 122(7): 1283-93.

28. Vold S, Ahmed IIK, Craven ER, Mattox C, Stamper R, Packer M, et al. Two-Year 
COMPASS Trial Results: Supraciliary Microstenting with Phacoemulsification in Patients with Open-Angle Glaucoma and Cataracts. Ophthalmology. 2016; 123(10):2103-12.

29. Issa SA, Pacheco J, Mahmood U, Nolan J, Beatty S. A novel index for predicting intraocular pressure reduction following cataract surgery. Br J Ophthalmol. 2005 May; 89(5):543-6.

30. Lin SC, Masis M, Porco TC, Pasquale LR. Predictors of Intraocular Pressure After Phacoemulsification in Primary Open-Angle Glaucoma Eyes with Wide Versus Narrower Angles (An American Ophthalmological Society Thesis). Trans Am Ophthalmol Soc [Internet]. 2017 Oct 23 [cited 2019 Mar 18];115. Available from: https://www.ncbi.nlm.nih.gov/pmc/articles/PMC5665659/

31. Zhou AW, Giroux J, Mao AJ, Hutnik CML. Can preoperative anterior chamber angle width predict magnitude of intraocular pressure change after cataract surgery? Canadian J ournal of Ophthalmology. 2010 J an 1;45(2): 149-53.

32. Poley BJ, Lindstrom RL, Samuelson TW, Schulze R. Intraocular pressure reduction after phacoemulsification with intraocular lens implantation in glaucomatous and nonglaucomatous eyes: Evaluation of a causal relationship between the natural lens and open-angle glaucoma. Journal of Cataract \& Refractive Surgery. 2009 Nov 1; 35(11):1946-55.

33. Wishart PK, Spaeth GL, Poryzees EM. Anterior chamber angle in the exfoliation syndrome. Br J Ophthalmol. 1985 Feb;69(2):103-7.

34. DeVience E, Chaudhry S, Saeedi OJ. Effect of intraoperative factors on IOP reduction after phacoemulsification. Int Ophthalmol. 2017 Feb;37(1):63-70.

35. Wang N, Chintala SK, Fini ME, Schuman JS. Ultrasound Activates the TM ELAM1/IL-1/NF-KB Response: A Potential Mechanism for Intraocular Pressure Reduction after Phacoemulsification. Invest Ophthalmol Vis Sci. 2003 May; 44(5):1977-81.

36. Bradley J M, Anderssohn AM, Colvis CM, Parshley DE, Zhu XH, Ruddat MS, et al. 
Mediation of laser trabeculoplasty-induced matrix metalloproteinase expression by IL-1beta and TNFalpha. Invest Ophthalmol Vis Sci. 2000 Feb; 41(2):422-30. 


\section{SUPPLEMENTARY MATERI ALS}

Supplementary Table 1: Univariate linear mixed-effects models of the clinical predictors of the intraocular pressure reduction following cataract surgery in PROGRESSA ( $\mathrm{N}=172$ eyes).

\begin{tabular}{|c|c|c|c|c|}
\hline $\begin{array}{l}\text { Variable used in the univariate } \\
\text { model }\end{array}$ & Estimate & $\begin{array}{l}\text { Standard } \\
\text { error }\end{array}$ & P-value & Pseudo- $\mathbf{R}^{2}$ \\
\hline Gender (male) & -0.83 & 0.44 & 0.06 & $2.7 \%$ \\
\hline Age at operation & 0.02 & 0.03 & 0.55 & $0.3 \%$ \\
\hline Maximum recorded IOP prior to operation & -0.13 & 0.04 & $1.1 \times 10^{-03}$ & $7.3 \%$ \\
\hline $\begin{array}{l}\text { Maximum IOP in the last } 2 \text { years prior to } \\
\text { cataract operation }\end{array}$ & -0.28 & 0.04 & $3.3 \times 10^{-10}$ & $22.5 \%$ \\
\hline Baseline study IOP & -0.05 & 0.04 & 0.18 & $1.3 \%$ \\
\hline Central corneal thickness & 0.00 & 0.01 & 0.69 & $0.1 \%$ \\
\hline Preoperative axial length† & -0.09 & 0.22 & 0.68 & $0.1 \%$ \\
\hline Preoperative anterior chamber depthł & -0.24 & 0.65 & 0.71 & $0.1 \%$ \\
\hline Pseudoexfoliation syndrome (yes) & -0.42 & 1.09 & 0.70 & $0.1 \%$ \\
\hline $\begin{array}{l}\text { Number of glaucoma medications } \\
\text { preoperatively }\end{array}$ & 0.84 & 0.23 & $4.4 \times 10^{-04}$ & $8.4 \%$ \\
\hline
\end{tabular}

Psuedo- $R^{2}$ is the variance explained by the fixed effects only of the generalized linear mixed-effects model. ${ }^{18}$

$\dagger$ Axial length was missing for 5 cases

‡ Anterior chamber depth was missing for 12 cases

IOP: intraocular pressure. 
Supplementary Figure 1: Cataract grading using the The Lens Opacities Classification System III of the cases and controls. This data was available for 153 cases and 164 controls.

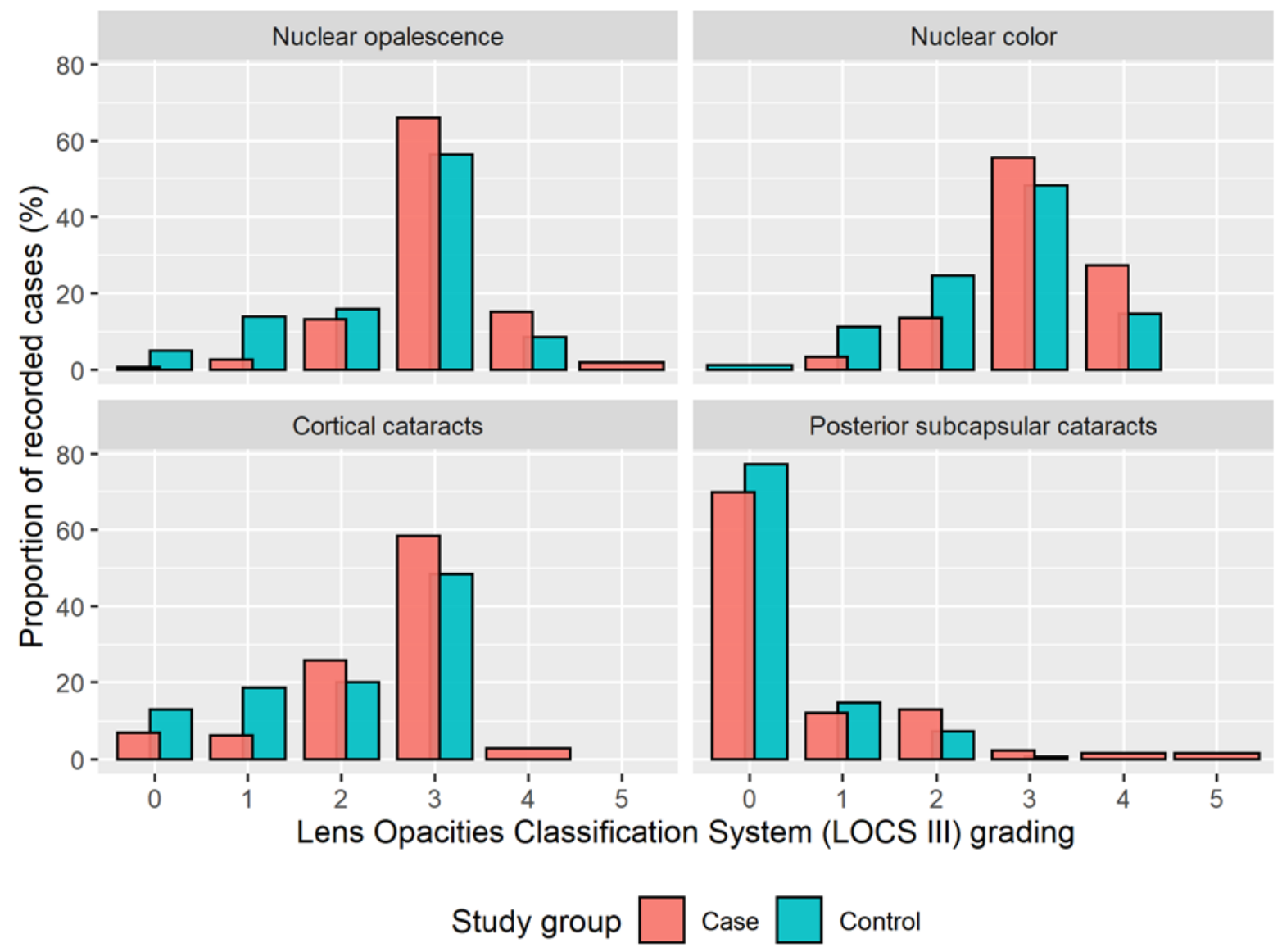


Supplementary Figure 2: Scatter plot of the preoperative anterior chamber depth and observed two-year mean change in intraocular pressure following cataract surgery.

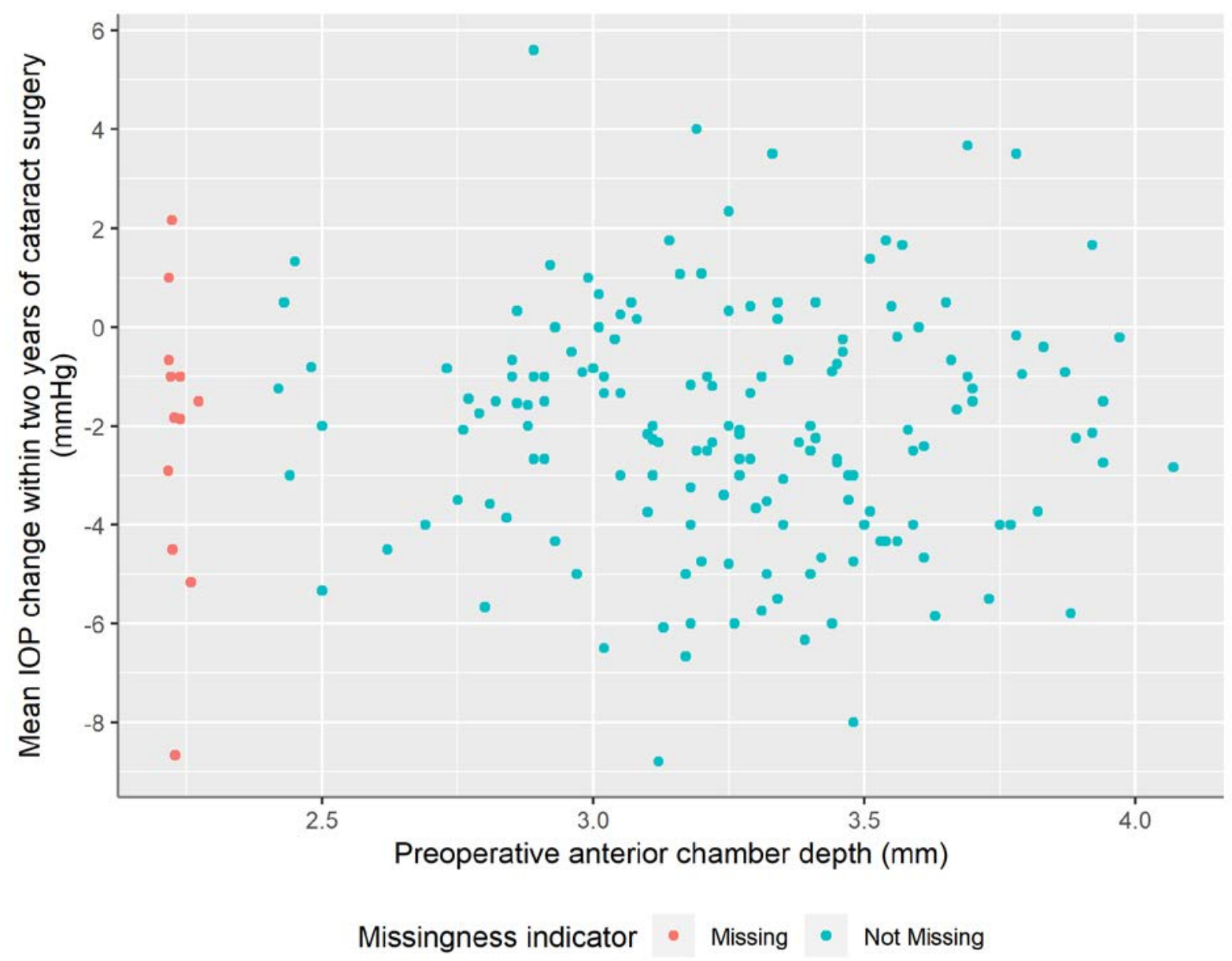

Each blue point represents an eye $(N=159)$. Red points $(N=12)$ are cases with missing preoperative anterior chamber depth. These are plotted on the left margin of the plot to assess for missingness bias. The missing cases appear to be missingat-random. 
Supplementary Figure 3: Boxplot of the observed two-year mean change in intraocular pressure following cataract surgery based on the number of preoperative glaucoma medications.

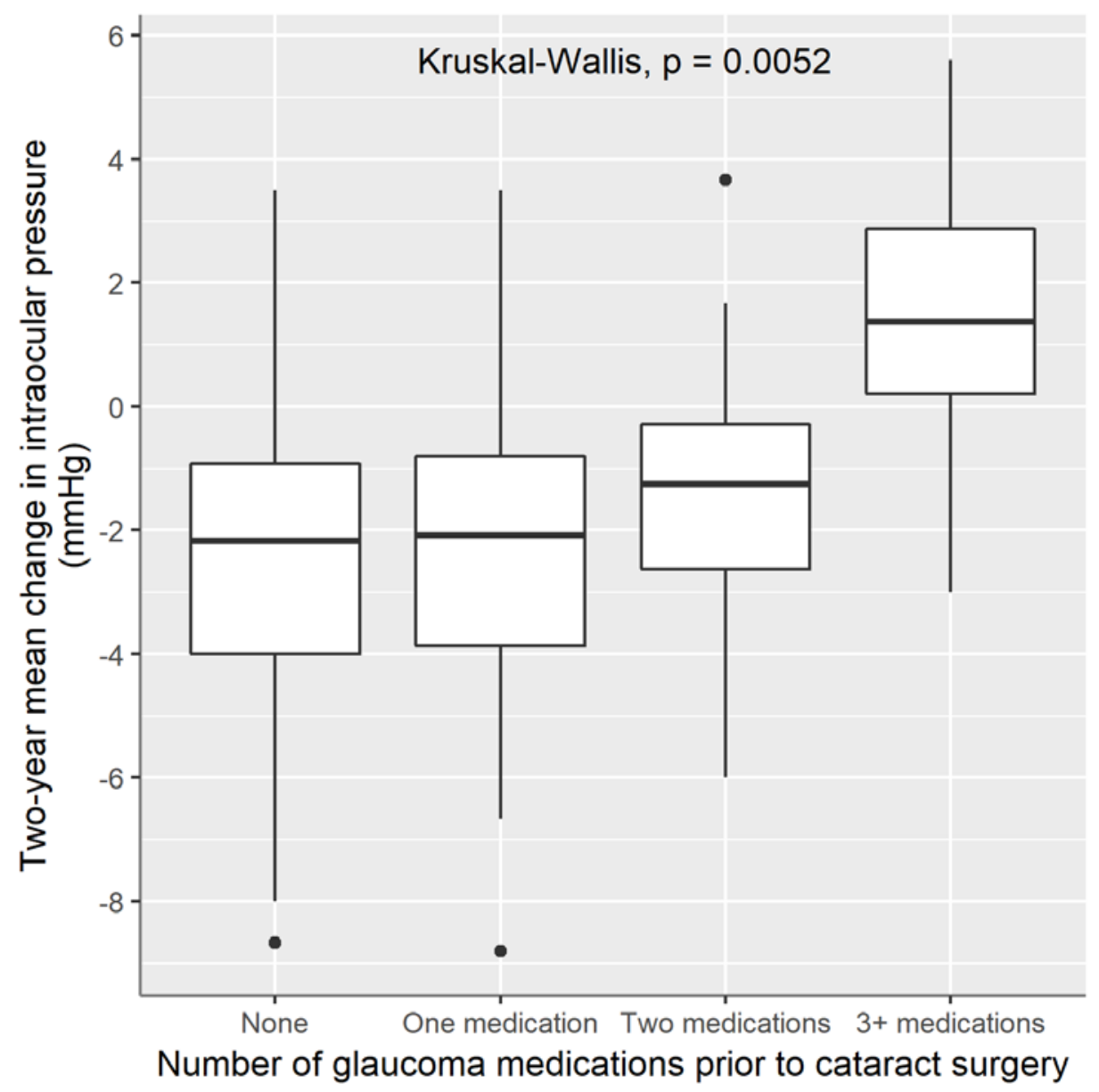

This article is protected by copyright. All rights reserved. 


\section{University Library}

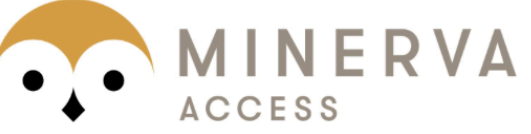

A gateway to Melbourne's research publications

Minerva Access is the Institutional Repository of The University of Melbourne

\section{Author/s:}

Qassim, A;Walland, MJ;Landers, J;Awadalla, M;Thi, N;Loh, J;Schulz, AM;Ridge,

B;Galanopoulos, A;Agar, A;Hewitt, AW;Graham, SL;Healey, PR;Casson, RJ;Craig, JE

Title:

Effect of phacoemulsification cataract surgery on intraocular pressure in early glaucoma: A prospective multi-site study

Date:

2020-02-13

\section{Citation:}

Qassim, A., Walland, M. J., Landers, J., Awadalla, M., Thi, N., Loh, J., Schulz, A. M., Ridge, B., Galanopoulos, A., Agar, A., Hewitt, A. W., Graham, S. L., Healey, P. R., Casson, R. J. \& Craig, J. E. (2020). Effect of phacoemulsification cataract surgery on intraocular pressure in early glaucoma: A prospective multi-site study. CLINICAL AND EXPERIMENTAL OPHTHALMOLOGY, 48 (4), pp.442-449. https://doi.org/10.1111/ceo.13724.

Persistent Link:

http://hdl.handle.net/11343/275408 